\title{
2-D Mapping of Ferroelectric Domains by Transmission Electron Microscopy
}

\author{
X.Q. Pan ${ }^{*}$, C. T. Nelson ${ }_{* * *}^{*}$ Y. Zhang ${ }^{*}$, S.J. Kim ${ }_{* * * *}^{*}$, B. Winchester ${ }^{* *}$, L.Q. Chen ${ }_{* * * * *}^{* *}$ A. Melville ${ }^{* * *}$, C.A.

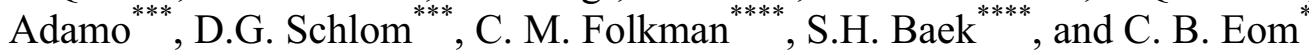 \\ *Department of Materials Science and Engineering, University of Michigan, Ann Arbor, MI 48109 \\ ** Department of Materials Science and Engineering, Penn State University, University Park, PA 16802 \\ ${ }^{* * *}$ Department of Materials Science and Engineering, Cornell University, Ithaca, NY 14853 \\ ${ }^{* * * *}$ Department of Materials Science and Engineering, University of Wisconsin-Madison, Madison, WI 53706
}

One of the key advantageous of ferroelectric materials is the vanishingly small critical size required for ferroelectricity as well as their narrow domain walls giving the potential for very high density data storage or use in confined geometries such as superlattices. Even at large length scales ferroelectric switching is dominated by nanoscale defects that modify the local energy density. For the common displacive ferroelectric materials, the determination of the local polarization can be accomplished by a direct interpretation of atom positions obtained by transmission electron microscopy (TEM) [1]. In this work we employ such methods to study the multiferroic $\mathrm{BiFeO}_{3}$ (BFO), a displacive perovskite ferroelectric. In particular we use the technique to study polarization spatial variation at the thin film interface and characterize polarization changes resulting from local ferroelectric switching.

The rhombohedral BFO is polarized along the $<111>$ directions (Fig. 1a) due to a shift of the oxygen octahedral and central Fe cation along the same axis which can be observed in TEM images. In this work (001) $\mathrm{BiFeO}_{3}$ films were grown on insulating (110) $\mathrm{TbScO}_{3}$ (TSO) single crystal substrates by molecular beam epitaxy. A subset of the films were grown on conducting $\mathrm{La}_{\mathrm{x}} \mathrm{Sr}_{1-\mathrm{x}} \mathrm{MnO}_{3}$ (LSMO) epitaxial buffer layers.

A small elongation along the polarization axis leads to four unique ferroelastic domains, $r_{1}-r_{4}$, illustrated in Fig. 1b according to the notation of Streiffer et al [2]. BFO grown on bare TSO forms a striped array of $r_{1}$ and $r_{4}$ ferroelastic domains with a $109^{\circ}$ polarization rotation due to misfit strain and depolarizing fields (Fig. 1b) [3]. Localized depolarizing fields are also created at the substrate interface as shown by the electrostatic energy plot in Fig. 1c according to our phase field model. Using the relative displacement of the B-site (Fe) cations to their 4 nearest A-site (Bi) neighbors we map the polarization within this region. Fig. $2 \mathrm{~b}$ shows an atomic resolution Z-contrast image of a pair of adjacent $109^{\circ}$ domain walls and Fig. 2c shows the corresponding cation displacement. We can see that the leftmost $109^{\circ}$ domain wall terminates at the substrate (Fig. 2d) but the local depolarizing fields at the right domain wall produce triangular $180^{\circ}$ reversed nanodomains (Fig. 2e). We show by phase-field modeling that these compensate the depolarizing fields and reduce the overall electrical energy of the film.

\section{References}

[1] C.L. Jia et al., Nature Materials, 6 (2007) 64.

[2] S.K. Streiffer et al., Journal of Applied Physics, 83 (1998) 2742.

[3] C.M. Folkman et al., Applied Physics Letters, 94 (2009) 251911.

[4] This work was supported through DOE grant DoE/BES DE-FG02-07ER46416, NSF grant DMR0820404 (YZ), and DOE grant DE-AC02-05CH11231 (NCEM user facilities). 
a

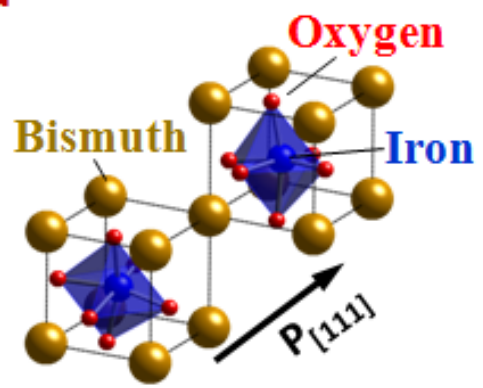

b

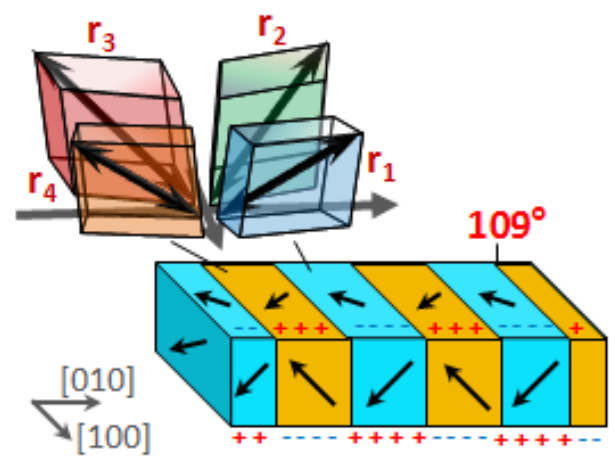

C

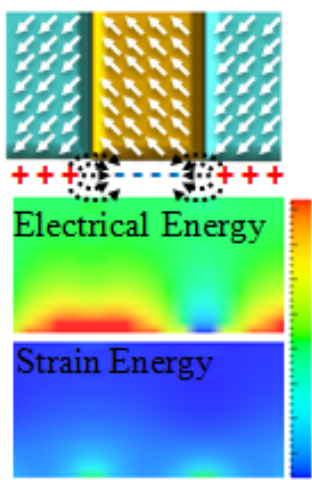

FIG 1. (a) Bulk rhombohedral $\mathrm{BiFeO}_{3}$ structure. (b) The four ferroelastic variants of the pseudocubic unit cell and a strain-induced periodic structure formed by twinning between $r_{1}^{-}$and $r_{4}{ }^{+}$ variants. (c) Phase-Field model of an unrelaxed $109^{\circ}$ domain structure showing an alternating highenergy (red) and low energy (blue) $109^{\circ}$ domain wall termination at the insulating substrate surface.

a

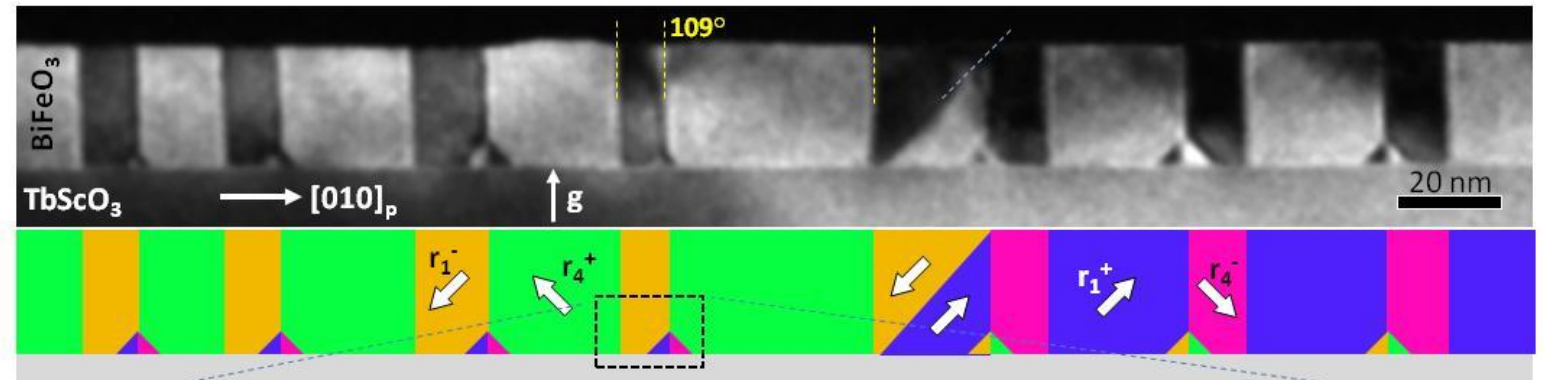

b
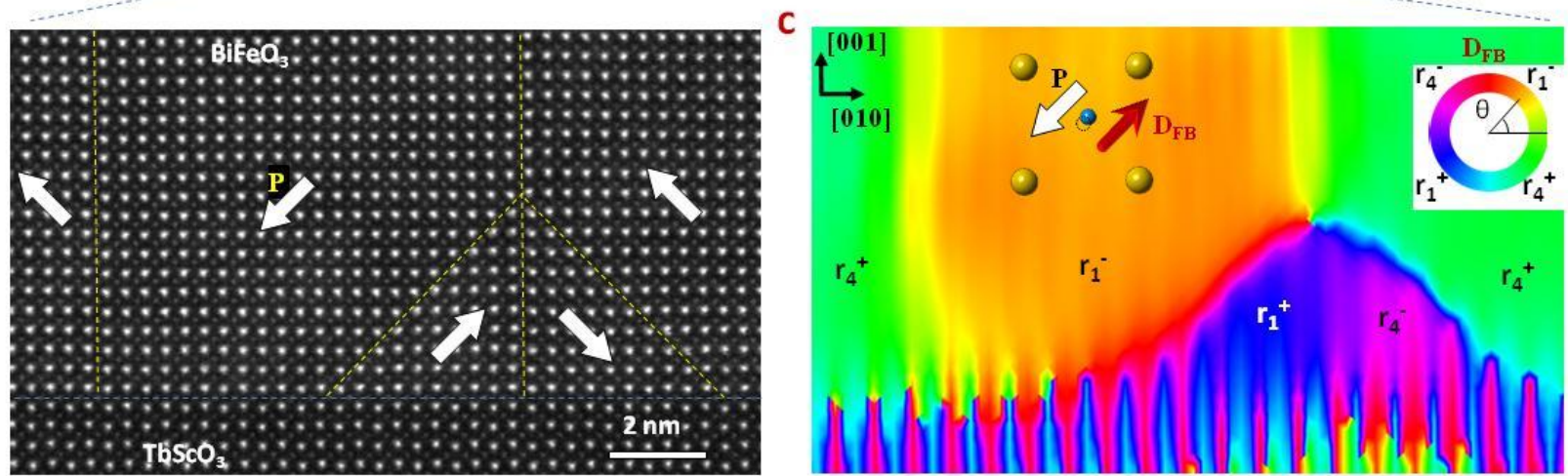

d
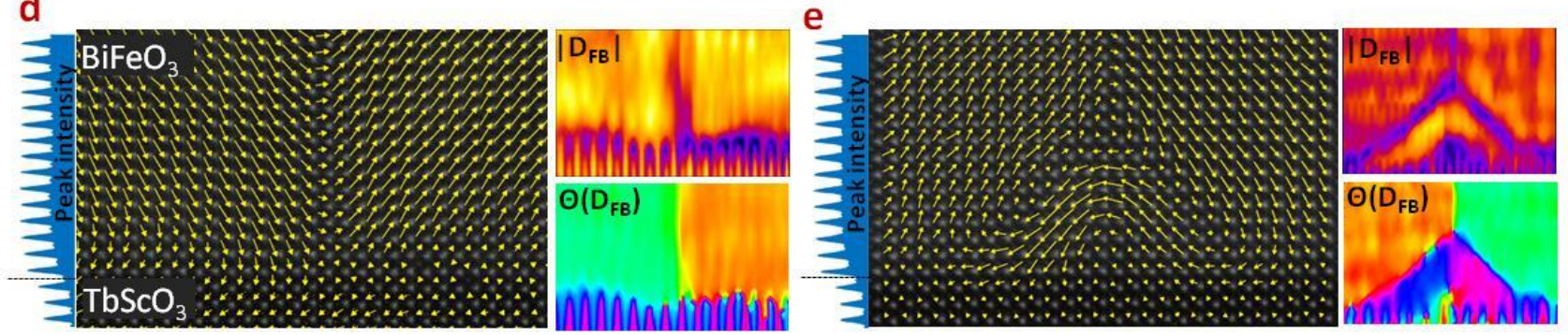

FIG 2 (a) Dark field TEM micrograph of the $109^{\circ}$ striped domain pattern of a $\mathrm{BiFeO}_{3}$ thin film. The domains variants are identified in the corresponding colored image. (b) Z-contrast image of a pair of adjacent $109^{\circ}$ domain walls and (c) the corresponding map of the Fe-Bi displacement $\left(\mathrm{D}_{\mathrm{FB}}\right)$ angle. (d) $\mathrm{A} \mathrm{D}_{\mathrm{FB}}$ vector map of the leftmost domain wall shows it terminates at the substrate surface. (e) A similar $\mathrm{D}_{\mathrm{FB}}$ vector map for the right domain wall illustrates the $180^{\circ}$ domains which form to compensate depolarizing fields resulting in a vortex with polarization closure domains. 